\title{
Balkanologie
}

Balkanologie Revue d'études pluridisciplinaires

Vol. X, $n^{\circ} 1-2 \mid 2008$

Volume $X$ Numéro 1-2

\section{Călăraşi et le Danube : une relation en demie-teinte entre la ville roumaine et le fleuve}

Odile Bratosin

\section{OpenEdition}

Journals

Édition électronique

URL : http://journals.openedition.org/balkanologie/389

DOI : 10.4000/balkanologie.389

ISSN : 1965-0582

\section{Éditeur}

Association française d'études sur les Balkans (Afebalk)

Référence électronique

Odile Bratosin, «Călăraşi et le Danube : une relation en demie-teinte entre la ville roumaine et le fleuve », Balkanologie [En ligne], Vol. X, n 1-2 | 2008, mis en ligne le 03 juin 2008, consulté le 17 décembre 2020. URL : http://journals.openedition.org/balkanologie/389 ; DOI : https://doi.org/ 10.4000/balkanologie.389

Ce document a été généré automatiquement le 17 décembre 2020.

(c) Tous droits réservés 


\title{
Călăraşi et le Danube : une relation en demie-teinte entre la ville roumaine et le fleuve
}

\author{
Odile Bratosin
}

1 Ville roumaine moyenne d'une région de grande culture, Călăraşi a la réputation d'être pauvre. La chute du régime communiste se traduit par une récessionéconomique et sociale. En dix ans, la ville perd $9 \%$ de sa population ${ }^{1}$. La fermeture progressive du complexe sidérurgique jette sur le marché du travail cadres et ouvriers qualifiés. Dans un rapport de l'Office des statistiques de la Commission européenne, à la fin de l'année 2001, Călăraşi figure, parmi 258 villes de l'Union européenne plus la Roumanie et Bulgarie, à la quatrième place pour son taux de chômage élevé avec 23,7 \%.

2 Călăraşi est avant tout une ville danubienne. A cet endroit, le fleuve se divise en plusieurs bras dont le Borcea qui baigne la ville et opère un changement de direction assez brusque en pénétrant en totalité en Roumanie. Ainsi son centre-ville, dans sa partie la plus à l'est, est riverain du Borcea et la périphérie sud de la commune est riveraine du Danube en amont de cette division. Hier le Danube, fierté des habitants de Călăraşi, magnifié dans les œuvres littéraires, servait pour le commerce et représentait une ouverture sur le reste du monde. Ainsi le port de Călăraşi était très actif, notamment pour le transport des céréales. Aujourd'hui la situation est paradoxale. L'ancien port de marchandises est quasi désert et le Danube reste le grand absent de la ville. Il constitue pourtant le seul élément sur lequel les habitants de Călăraşi s'accordent pour reconnaître qu'il est un atout pour la ville.

3 Ceci dit, à Călăraşi, le Danube, fleuve européen par excellence et frontière entre la Roumanie et la Bulgarie, est l'objet de plusieurs projets transfrontaliers et de coopérations entre les deux pays, projets dont les instances européennes suscitent fortement l'émergence et $\mathrm{y}$ apportent une aide financière.

4 Se trouve t-on devant une situation où le fleuve échappe à ses riverains et est approprié par les institutionnels? La politique de développement régional et de coopération transfrontalière de l'Europe est-elle adaptée aux anciens pays communistes d'Europe 
orientale pour générer un développement équilibré et durable tout en répondant aux besoins des populations? L'étude du cas de Călăraşi et de ses relations avec sa voisine bulgare, Silistra, nous donne un aperçu du chemin à parcourir et des spécificités locales à prendre en compte.

Après un rappel du rôle joué par le Danube comme point d'ancrage de la ville de Călăraşi et comme ressource vitale, nous analyserons les modalités du développement local et régional et leurs répercussions sur la place du fleuve dans la ville.

\section{Le paradoxe d'une ville dont l'histoire la conduit au Danube mais dont le plan d'urbanisme l'en éloigne}

\section{Les origines danubiennes de la ville}

\section{Une halte sur la route Bucarest-Istanbul}

6 C'est au Danube que Călăraşi doit son existence en tant que ville. À l'origine le village de Lichireşti ( $1^{\text {ère }}$ attestation juridique datée du 25 juillet 1630) est une halte, avant la traversée du fleuve, pour les commerçants qui se rendent à Silistra, chef-lieu de province de l'Empire ottoman, puis à la fin du XVIIème siècle, pour les coursiers militaires appelés "Călăraşi ştafetari"2 qui assurent le service du courrier de Bucarest à Istanbul. Après leur carrière militaire, ces derniers s'installent sur place donnant ainsi leur nom à la ville. Les bases des premières structures administratives de la cité peuvent être datées du début du XVIII ${ }^{\text {mèe }}$ siècle par la présence d'un poste de douane comme en témoignent les documents d'archives ${ }^{3}$.

\section{Le Danube « nourricier »}

7 Le Danube est la raison d'être des premiers occupants de cette région et en accompagne le développement. La richesse et l'abondance de la faune et de la flore favorisent dès le néolithique l'occupation des berges du Danube par des communautés humaines. Pêche, chasse, élevage, agriculture, apiculture et récolte des roseaux sont les principales ressources des premiers occupants du site. La littérature roumaine met en évidence la faune, la flore, les paysages de forêts et de marais, autant de symboles de la vie qui anime la vallée du Danube. Celle-ci est décrite par contraste avec la plaine désertique du Baragan, longtemps inculte. Il faut attendre la fin du XIXème siècle avant de constater une mise en valeur progressive du Baragan par le creusement de puits et, à partir de $1961^{4}$, l'aménagement du Danube permet le développement de l'irrigation et de la culture intensive des céréales.

\section{Le Danube « protecteur »}

8 Entre le $6^{\mathrm{e}}$ et le $4^{\mathrm{e}}$ siècle av. J.-C, le danger scythe dans la région du Bas Danube et la proximité avec les colonies grecques de la mer Noire conduisent une partie de la population gète de la rive gauche du Borcea sur la rive droite du Danube. Cette migration serait à l'origine de la série de couples de villes sur la frontière roumanobulgare ${ }^{5}$. Le Danube joue à nouveau un rôle protecteur pendant la période des invasions barbares, slaves, bulgares, mongoles, qui débutent au $3^{\mathrm{e}}$ siècle et durent près de 1000 ans. Les populations se réfugient sur les îles, plus nombreuses qu'actuellement comme 
en atteste une carte autrichienne de $1791^{6}$ et traversent le Danube d'une rive à l'autre. Ces mouvements se poursuivent au XIX ${ }^{\text {ème }}$ siècle pendant les guerres russo-turques. Dès 1769, Călăraşi est le théâtre des guerres entre Russes et Turcs. Dans les années 1810-1812, les opérations militaires sont de plus grande envergure, Silistra est assiégée et détruite pour une grande partie. Ses habitants doivent se réfugier sur la rive gauche du Danube. Le deuxième exode de 1836 correspond au départ des Russes de Silistra et à la remise de la ville aux autorités ottomanes. Un grand nombre d'habitants de Silistra s'installe alors définitivement à Călăraşi.

\section{Un élément fort de l'identité culturelle locale}

9 Cette occupation ancienne par une même population qui vit indifféremment sur les deux rives du Danube a scellé une culture et une identité propres à la vallée danubienne. Cet héritage culturel se découvre aujourd'hui dans les sites archéologiques et dans le patrimoine architectural des deux villes. La littérature roumaine de cette région abonde en référence au Danube. Dans la poésie et les chants folkloriques, il fédère l'histoire personnelle à l'histoire collective. Il rappelle l'occupation turque de la rive droite, vécue douloureusement pas la population de la rive gauche. Ainsi dans le chant épique "Cântecul Ilencutei"7 (Le chant d'Ilencuta), le Danube apparaît comme lieu de mort et de salut en même temps. Ilencuta, enlevée par les Turcs et amenée ligotée sur un bateau, ruse pour se jeter dans le Danube; elle préfère mourir noyée dans le Danube que de vivre en esclave chez les Turcs.

Dans un autre registre, les écrits retracent aussi les histoires qui jalonnent les trafics et le commerce entre les deux rives. Les textes du XIX ${ }^{\text {ème }}$ et du début du XX ${ }^{\text {ème }}$ siècle mentionnent le Borcea pour indiquer la position géographique de la ville de Călăraşi et le Danube est représenté comme un élément du paysage et de la vie économique. Certains auteurs, comme Alexandru Vlahuta, présentent le Danube comme le bras unificateur des "terres ondulées de Dobroudja" et de "l'interminable plaine du Baragan"8. Il a été l'axe de développement de cette région malgré la frontière édifiée par les pouvoirs politiques. Il est le lien avec une Europe qui paraît lointaine à cette région excentrée. De plus, il est le symbole d'un pays européen qui a connu cinq siècles de soumission à l'Empire ottoman et qui souhaiterait que soit reconnue son identité européenne. Encore aujourd'hui, le fleuve est considéré comme le symbole d'une appartenance et d'une participation à la construction de l'Europe, Cătălin ţârlea, écrivain contemporain, déclare que "Călăraşi est une grande ville danubienne, le Danube étant une grande entité de l'Europe. Par conséquent, Călăraşi est une ville européenne "9.

11 Cette relation au Danube va progressivement se modifier avec l'urbanisation des lieux tout en restant très fonctionnelle.

\section{Le développement urbain de Călăraşi l'éloigne du fleuve}

\section{Le carcan du régime féodal}

Il faut attendre la fin du XVIII ${ }^{\text {ème }}$ siècle pour que la cité de Călăraşi connaisse les premiers changements notables. La construction de quelques édifices à caractère public est rendue nécessaire par la multiplication des échanges entre Călăraşi et Silistra. Il s'agit d'une quarantaine où sont soumis à un contrôle sévère tous ceux qui viennent de 
Silistra et d'une auberge pour héberger voyageurs et commerçants affluant dans la cité. Jusqu'à la moitié du XIX ${ }^{\text {ème }}$ siècle, Călăraşi ressemble encore à un gros bourg d'une région agricole. Le monastère ColŢea de Bucarest, puis hôpital Colțea, possède de nombreux terrains à Călăraşi. Il prélève la dîme sur les récoltes, l'eau de vie et les produits de la vigne et de la pêche, mais ne se contentant pas des revenus du domaine, de nombreux conflits surviennent entre les administrateurs du domaine et les habitants. Cette domination est à l'origine du retard de développement de la cité et les tensions qui l'accompagnent occasionnent des demandes soutenues et répétées de libération de la ville.

\section{Une urbanisation tardive}

Le développement urbain résulte du changement de statut en 1833, la ville est élevée au rang de chef-lieu de judeȚ (département). La désignation de Călăraşi comme chef-lieu du judeŢ Ialomiţa entraîne l'augmentation des échanges commerciaux vers les grands centres européens via le Danube et l'installation d'une bourgeoisie. La transformation de la cité s'impose. Alors que sa population stagnejusqu'en1833, de 738 habitants en 1775 , elle n'atteint que 860 habitants en 1833, celle-ci augmente très rapidement ensuite; en 4 ans elle double presque, passant à 1605 habitants en $1837^{10}$. L'urbanisation est planifiée selon un schéma s'inspirant du processus d'alignement et d'organisation destiné aux localités rurales dans les années 1830-1840. Il se présente sous la forme d'un quadrillage de parcelles rectangulaires qui découpe la ville. Les exigences financières du monastère de ColŢea freinent les ventes de terrain, il faut attendre 1852 et le soutien du prince régnant Barbu Stirbei pour qu'il donne une nouvelle impulsion au développement de la ville par ses conseils.

14 Les relations vitales, agricoles et commerciales que la population entretient avec le Danube et le Borcea vont alors, petit à petit, se relâcher. D'une bourgade rurale, à proximité des berges du Danube, Călăraşi devient peu à peu une ville qui, pour se protéger des inondations, va privilégier une position à l'ouest et en retrait du Borcea et du Jirlăul, l'ancien bras du Danube.

\section{Un développement urbain rapide et planifié}

L'implantation initiale de la population correspond à l'actuel quartier Magureni. Le village est construit sur la berge du Borcea face à l'île, d'où une très grande proximité avec le fleuve. Entre 1833 et 1851, la ville est encore bâtie sur le Borcea de part et d'autre du port. Les premières extensions sont décidées en 1851 d'après le plan de l'ingénieur Scarlat Popovici ${ }^{11}$ (figure 1). Celui-ci conserve un maximum de constructions mais la parcellisation standardisée ne tient aucunement compte du foncier existant et de l'identité de la localité. La liaison entre la ville et son fleuve n'est pas rompue, elle est avant tout fonctionnelle, tout comme dans d'autres villes commerçantes et/ou industrielles du XIX ${ }^{\text {ème }}$ siècle. 
Figure 1. Plan de 1851 et en fond de plan le parcellaire d'origine

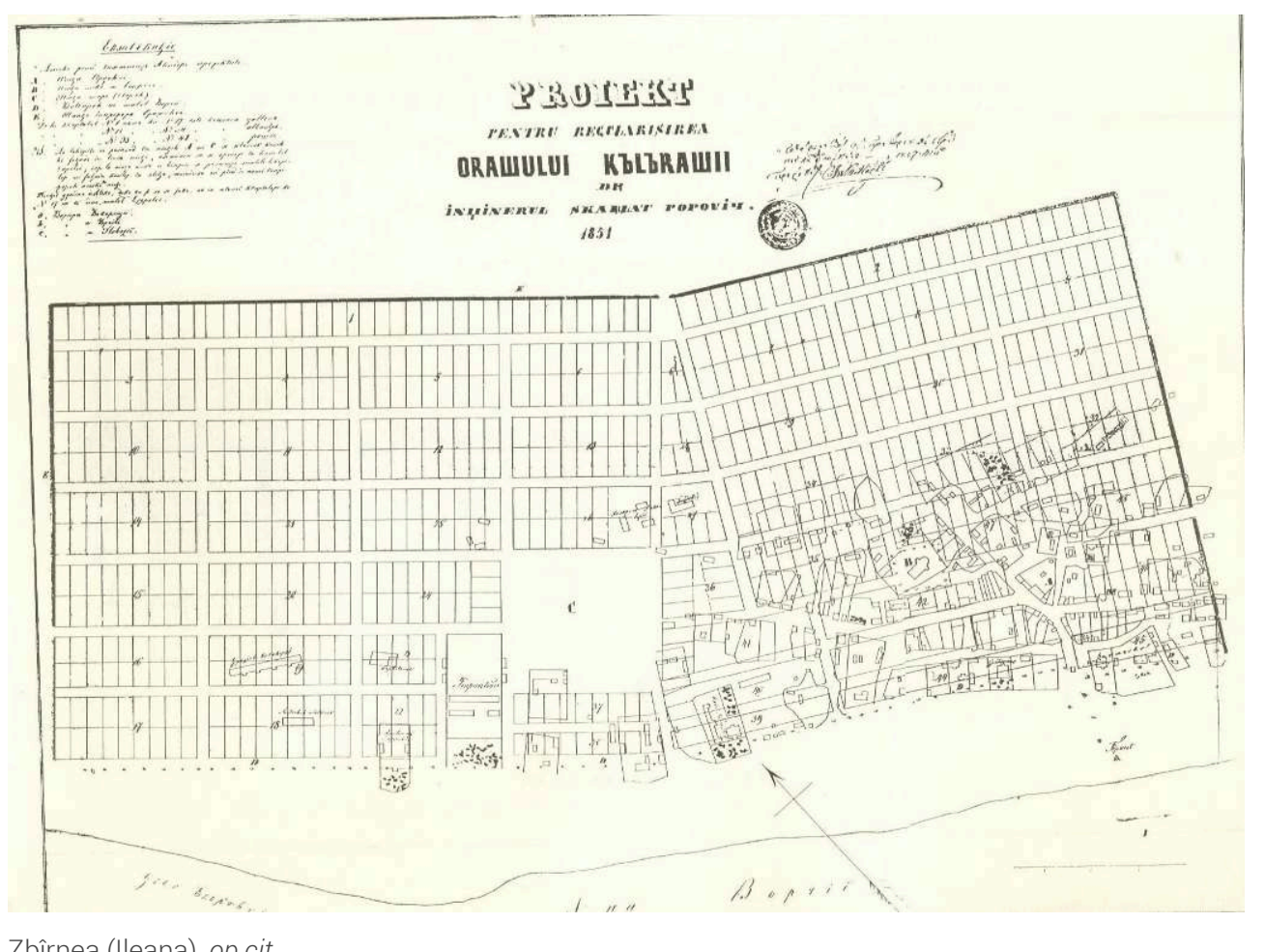

La croissance démographique s'accélère depuis 1833 à des rythmes annuels de l'ordre de 4 à $5 \%$ par an. Ainsi, en 1859 on dénombre 3497 habitants et 7734 habitants en 1887. Ce dynamisme dépasse les prévisions de développement de la ville et 24 ans après, celle-ci esttrop à l'étroit dans ses limites de 1851. De nouvelles extensions sont décidées en 1875,1888 et $1925-1926^{12}$. L'extension de 1925-1926 est la plus importante, quartiers d'habitations à l'ouest et au nord, à l'est la modernisation de la zone portuaire avec l'arrivée de la voie ferrée sur le port, fait de Călăraşi une ville portuaire. Le port, à l'activité importante à cette époque avec le transport de céréales du Baragan et de bétail, assure une jonction entre la ville et son cours d'eau dans la continuité de celle-ci. Il est un lieu de vie et d'échanges avec le monde extérieur. Il symbolise le dynamisme économique de la ville, fait figure de vitrine, représenté sur les cartes postales sous plusieurs vues. De part et d'autre du port, l'espace dédié aux activités se prolonge avec plusieurs équipements publics d'intérêts économiques: usines d'eau et d'électricité, abattoir.... L'espace réservé à la promenade tient dans un jardin public alors bordé par un bras du Borcea servant de champ d'expansion des crues.

En $1911^{13}$, des cartes militaires allemandes montrent l'installation de deux régiments et la mise en relation des villages de Mircea Voda et de Magureni avec Călăraşi. On peut donc considérer qu'avant 1930 la ville avait une extension proche de ce qu'elle est actuellement, dès lors l'orientation est-ouest de l'extension de la ville s'affirme et la relation au fleuve s'oublie. La période communiste ne comporte pas d'innovation mais une poursuite et une accélération de l'industrialisation qui fige définitivement la morphologie de la ville entre une zone industrielle à l'est au-delà de la voie ferrée de 1962 et, à l'extrémité ouest de la ville, le début de la construction en 1976 du combinat sidérurgique. Ces deux ensembles forment un rempart à l'extension de l'habitat qui se développe essentiellement à l'ouest entre le combinat et les anciens quartiers d'habitat 
individuel. Ainsi, des quartiers d'habitations collectives à forte densité sont érigés entre les années 1960 et 1980, puis terminés dans les années 1990. Depuis, de nouvelles constructions collectives et individuelles sont réalisées dans les espaces vides.

Figure 2. Plan récent de Călăraşi

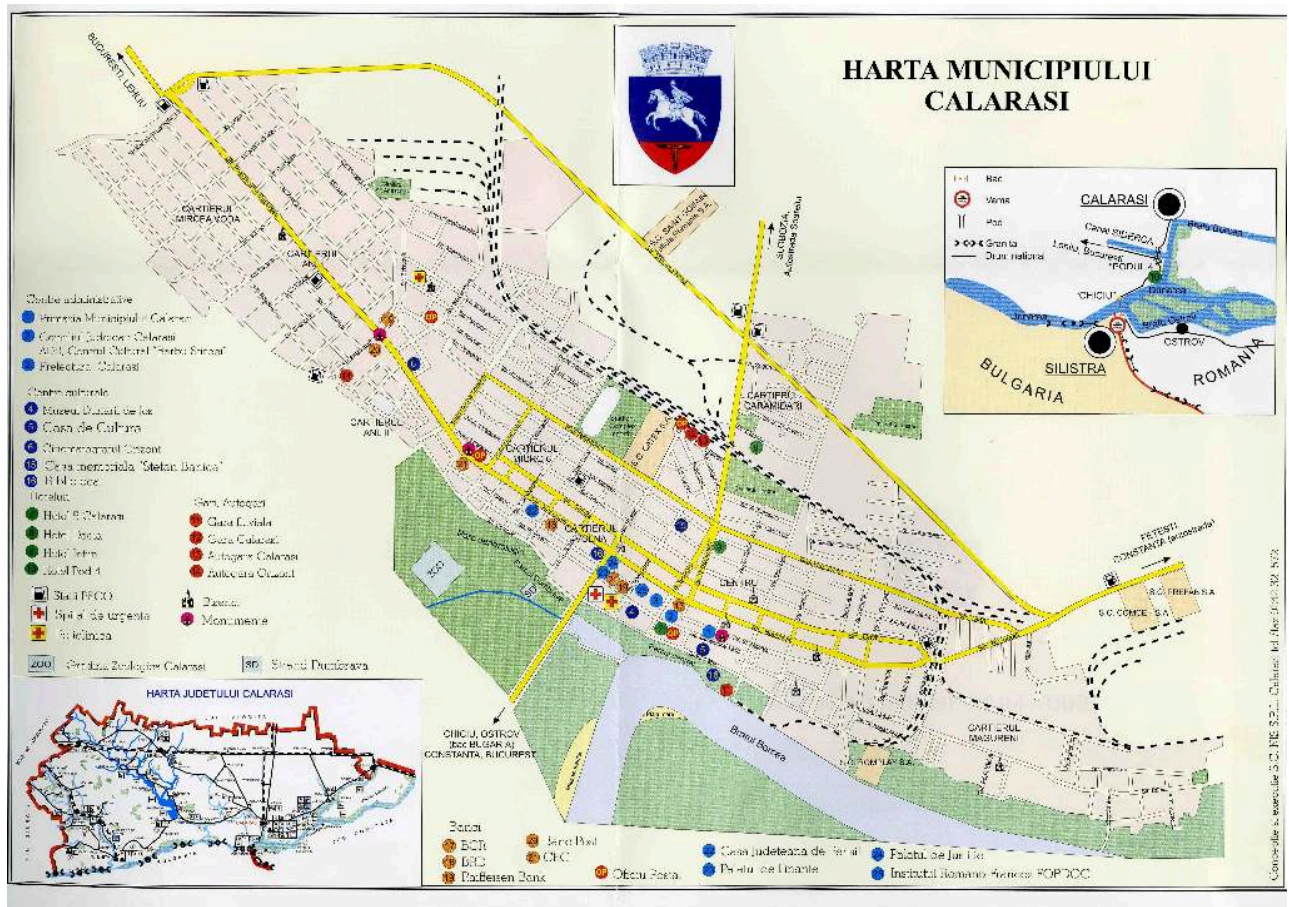

Primăria Municipiului Călăraşi, carte accompagnant un DVD offert par Monsieur le maire

\section{Un schéma urbain et fonctionnel qui ignore le fleuve}

La morphologie du plan d'urbanisme adopté au XIX ${ }^{\text {ème }}$ siècle et l'industrialisation de la ville participent à l'éloignement progressif de la ville de son fleuve. Toutes les extensionsrespectent la géométrie orthogonale du plan de 1851. Celui-ci a le défaut d'être peu sinueux et de proposer des voies perpendiculaires au cours d'eau qui débouchent sur celui-ci, ou plutôt, pour certaines en tant qu'impasse, ne l'atteignent jamais. Enserrée entre deux infrastructures de communication, la voie ferrée au nord et le Borcea au sud, la ville va se développer en longueur sur son axe est-ouest. Ce schéma tend à canaliser les flux de circulation selon cet axe parallèle au Borcea, limitant les déplacements vers celui-ci. Par conséquent, aucun itinéraire, ni de vue directe depuis le centre-ville sur le fleuve, ni de front d'eau constitué d'immeubles d'habitations n'existent. Pire encore, ces formes urbaines privilégiant le carré ou le rectangle sont "porteurs de projets réfléchis de conquête et d'occupation de l'espace" que décrit Guy Di Méo ${ }^{14}$ et ne permettent pas d'évolution de la trame urbaine. Pour preuve, depuis l'origine de l'urbanisation du site, aucune transformation radicale du parcellaire n'a été tentée. On le voit encore aujourd'hui, un étranger arrivant dans la ville par la voie principale, rue Bucarest, peut ignorer complètement le fleuve puisque ses pas ne sont pas détournés de l'artère principale.

Actuellement la population se trouve dans une situation où les aménagements successifs l'ont privée du Borcea qui n'est visible que du jardin public et de l'ancien 
port d'où l'on peut embarquer vers la plage située sur l'île face à la ville. En effet, l'accès au fleuve est restreint à une petite section du Borcea, la route menant à Chiciu et à la Bulgarie forme une digue et a définitivement asséché lejirlăul, cet autre bras du Danube. L'implantation d'une zone industrielle au-delà de la voie ferrée déporte vers l'est de la ville le transport de marchandises, essentiellement des pondéreux, et isole cette portion de rive du centre-ville historique privant la population d'un cheminement continu le long de la berge. Le quartier Magureni qui fut la première zone d'implantation de la population de Călăraşi, se trouve, de ce fait, complètement coupé du reste de la ville et conserve encore un aspect rural (figure 4).

Figure 3. Vue d'une zone d'activités aménagée entre la voie de chemin de fer et le quartier Magureni au fond.

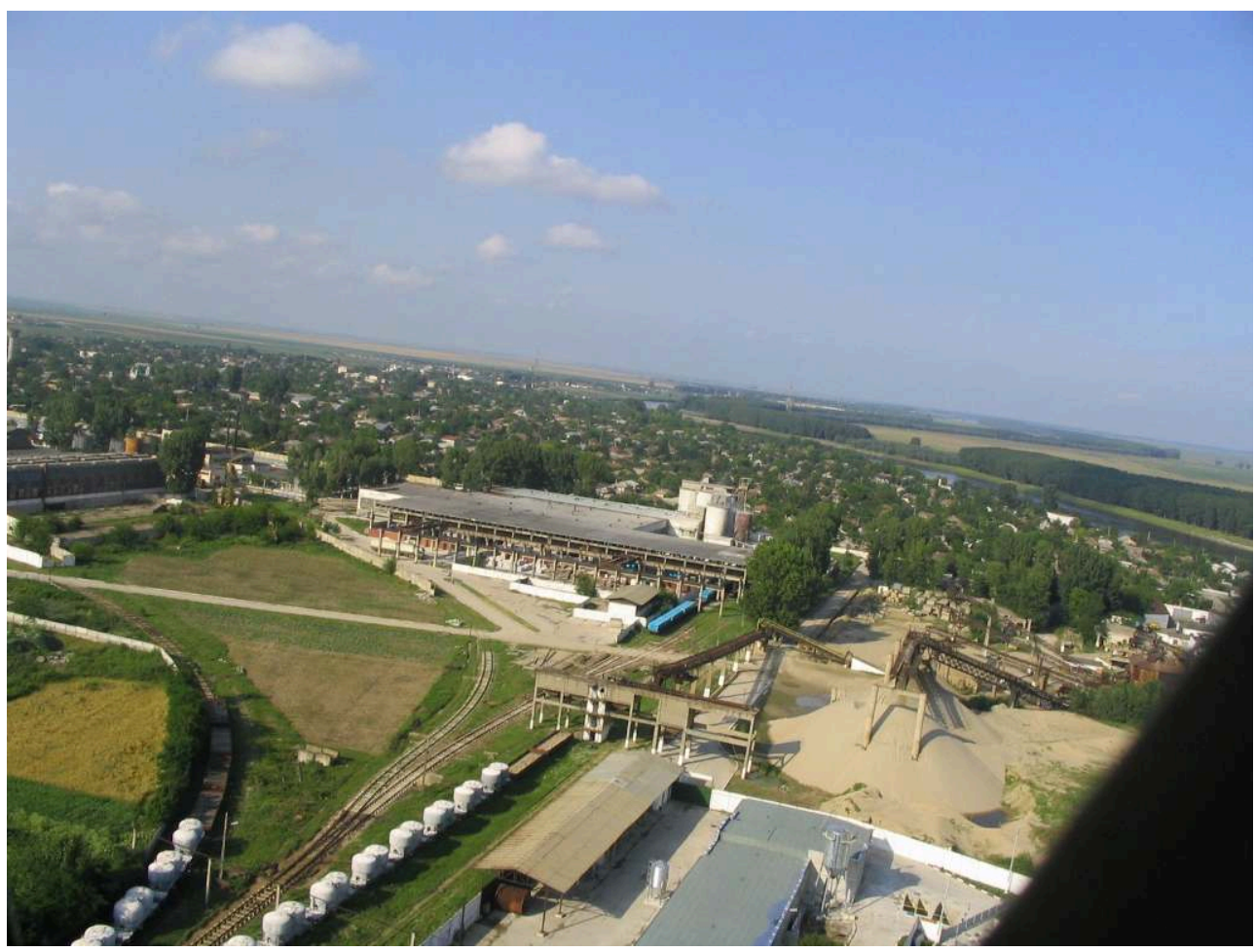

Commentaire de la photo : La proximité du Borcea et de la voie ferrée ont été certainement des arguments de poids dans le choix de ce site, l'entreprise que l'on distingue utilise effectivement ces deux moyens de transport pour relier le reste de la Roumanie via le rail ou le reste de l'Europe via la voie d'eau Cette implantation a entraîné une coupure durable de l'espace marqué par un mur d'enceinte qui isole davantage encore le quartier Magureni de la ville, contraint à une vie villageoise. La berge du Borcea n'est plus accessible depuis la ville et elle est réservée à une utilisation industrielle

Photo prise en 2004 par Florin Rădulescu, chargé de mission du patrimoine à la Direction départementale de la culture, du patrimoine et des cultes.

Le manque d'entretien régulier du jardin public et la présence d'un bassin de rétention, ajouté aux difficultés d'accès et à la faible intégration du Borcea dans la ville, ne rendent pas très attractives les rives du fleuve malgré les liens qui le rattachent à la population. Les souvenirs d'enfance, la littérature, l'histoire locale, la beauté du fleuve et de ses berges, sont souvent cités comme les seuls lieux agréables et calmes de la ville.

Dans les pays développés, la période post-industrielle est marquée par le phénomène de désindustrialisation laissant apparaître des friches en ville, elle correspond à la phase de déclin dans le "cycle d'évolution des relations ville fleuve" ${ }^{15}$. Une réaction s'amorce dans les années 1980-1990 au cours desquelles de nombreux projets d'aménagement de 
front d'eau concernent des territoires industriels en mutation. Ainsi en France, la ville de Nantes développe le concept de "l'île de Nantes au coeur de l'agglomération"16 et Lyon le projet "Lyon-confluence" ${ }^{17}$. Plus qu'une redécouverte du potentiel poétique, ce mouvement de retour de la ville vers son fleuve désigne une volonté de réappropriation du fleuve par ses riverains et d'accroissement du rayonnement de la ville. A l'inverse, Călăraşi est toujours dans une phase d'industrialisation: Saint-Gobain y a ouvert une usine en février 2006 et la ville privilégie la fonctionnalité dans ses relations au Danube. Cette position est à replacer dans le contexte historique de la Roumanie et de la ville. Călăraşi est à un moment clef de son histoire dans cette phase transitoire avant l'entrée du pays dans l'Union européenne. Après avoir connu, dans les années 1990, une période de récession, les années 2000 marquent un renouveau économique. La modernisation des institutions vers la démocratie et la mise en pratique du principe européen de subsidiarité donnent un nouveau cadre au développement économique. Il ne suit plus un chemin descendant dans lequel le pouvoir central imposait ses directives aux autorités locales. Celles-ci ont maintenant un rôle majeur en terme d'initiative, la Préfecture est un acteur parmi d'autres, elle est promoteur du développement local et contrôle les décisions locales. L'obligation de constituer des collectivités capables de gérer les fonds de pré-adhésion et l'incitation de l'Union européenne à la création de régions de coopération transfrontalière ajoutent des territoires d'action à ceux du judeT et de la commune et multiplient le nombre d'acteurs. Le texte qui suit est empreint de cette nouvelle situation. Notre démarche repose sur une analyse multi-niveaux et multi-acteurs des rapports de la ville à son fleuve au travers du prisme des projets de développement et d'aménagement.

\section{Le Danube en tant que lieu de vie des habitants de Călăraşi n'est pas la préoccupation du moment}

\section{Une stratégie de développement qui reste lettre morte par manque de portage politique}

La phase préparatoire à l'intégration européenne s'accompagne d'aides financières importantes pour permettre aux pays candidats de rejoindre le niveau de développement despays de l'Union européenne. Le long chemin à parcourir demande une mobilisation générale des autorités administratives, tant nationales que locales, et des élus locaux. On trouve trace de cette mobilisation dans de nombreux documents de programmes au niveau national, régional et départemental mais la recherche de résultats concrets ne mène qu'à des réponses évasives faisant référence aux documents précités ou même à l'aveu d'absence de réalisations. Les écrits des journaux reflètent cette situation, les termes restent flous se limitant à des présentations générales d'objectifs à atteindre. Les étapes d'initiation des projets, signature d'accords ou obtention de financement, sont relatées dans les hebdomadaires de Călăraşi mais rarement les résultats concrets obtenus et les expériences développées. A tous les niveaux locaux de décision le même phénomène s'observe, qui tend à donner une image chaotique et conduit au marasme des affaires du judeţ et de la ville de Călăraşi. 


\section{Les lacunes de la coopération entre les Etats roumain et bulgare}

24 $\mathrm{du} \mathrm{XX}^{\mathrm{ème}}$ siècle. La faible coopération entre les deux pays, que l'on observe actuellement, date du communisme, quand la Bulgarie avait des relations préférentielles avec l'URSS, contrairement à la Roumanie. La chute du régime communiste n'a pas modifié la situation car "pour rejoindre le marché économique occidental et la démocratie ils ont opté pour une stratégie individuelle et non commune"18. Attitude d'autant plus pénalisante pour les deux pays qu'elle engendre une concurrence entre eux, la Roumanie, dans une position plus centrale en Europe adopte une attitude de supériorité, se considérant plus riche que la Bulgarie. Opposition que le débat politique européen entretient avec la comparaison des niveaux d'avancement des deux pays dans les négociations d'adhésion ${ }^{19}$. L'objectif "d'aboutir à un système transfrontalier intégré dans un cadre supranational"20 par un renforcement de la coopération régionale ne semble pas être celui de nombreux élus. Le Danube qui autrefois servait de pont entre les deux rives devient le support de leur différence. L'aménagement des berges du fleuve en témoigne, jardin bien entretenu et hôtel de luxe à Silistra, jardin et zoo quelque peu négligés, parc touristique et d'affaires en souffrance à Călăraşi par absence de portage politique et financier. A l'aube de l'entrée dans l'Union européenne de ces deux pays voisins et à l'heure de la disparition des frontières en Europe, la construction d'un nouveau poste frontière entre Călăraşi et Silistra sème le doute sur leur volonté de coopérer. Annoncé comme devant améliorer les échanges commerciaux entre les deux rives, chacun publie une date de réalisation différente et l'actuel président du conseil de județ de Călăraşi accuse les Bulgares d'être les responsables du retard. Le projet bulgare, non abouti, de construction d'une centrale nucléaire à $107 \mathrm{~km}$ au sud-ouest du judeţ Călăraşi, menaçant directement les villes roumaines de la vallée du Danube, est un autre exemple d'absence de coopération au niveau des Etats.

\section{La région de développement sud Muntenia n'est pas appropriée par les élus}

Pour se conformer à la politique européenne de développement dont le cadre opératoire est la région, le Parlement roumain a adopté en 1998 la loi 151/98 sur le développement régional dont l'une des mesures a introduit un découpage en huit régions. Elle intervient après une période de centralisation du pouvoir décisionnel qui faisait elle-même suite à une démocratie trop jeune pour avoir pu développer une culture politique locale. La réforme de la représentation locale n'est pas achevée, elle se heurte encore au « projet idéologique de la nation unitaire $»^{21}$. La loi 151/98 ne prévoit pas de régions administratives territoriales mais des régions de développement permettant à l'Union européenne de verser des fonds pour des projets sélectionnés. L'Agence Nationale de Développement Régional transformée en Plan National de Développement (PND) directement rattachée au Ministère du Développement et de la Prévision (MDP) et les Agences de développement régional (ADR), perçoivent ces fonds et les redistribuent. Les Agences régionales ont le statut d'organisations non gouvernementales dont les membres sont nommés par les autorités. Leur rôle est de servir d'interface entre le PND d'une part, les collectivités territoriales et les

Balkanologie, Vol. X, n 1-2 | 2008 
entrepreneurs d'autre part. En réalité leur rôle se limite à opérer une présélection des dossiers de demandes d'aides européennes, la décision finale relevant du MDP.

Figure 4. Les régions de développement roumaines

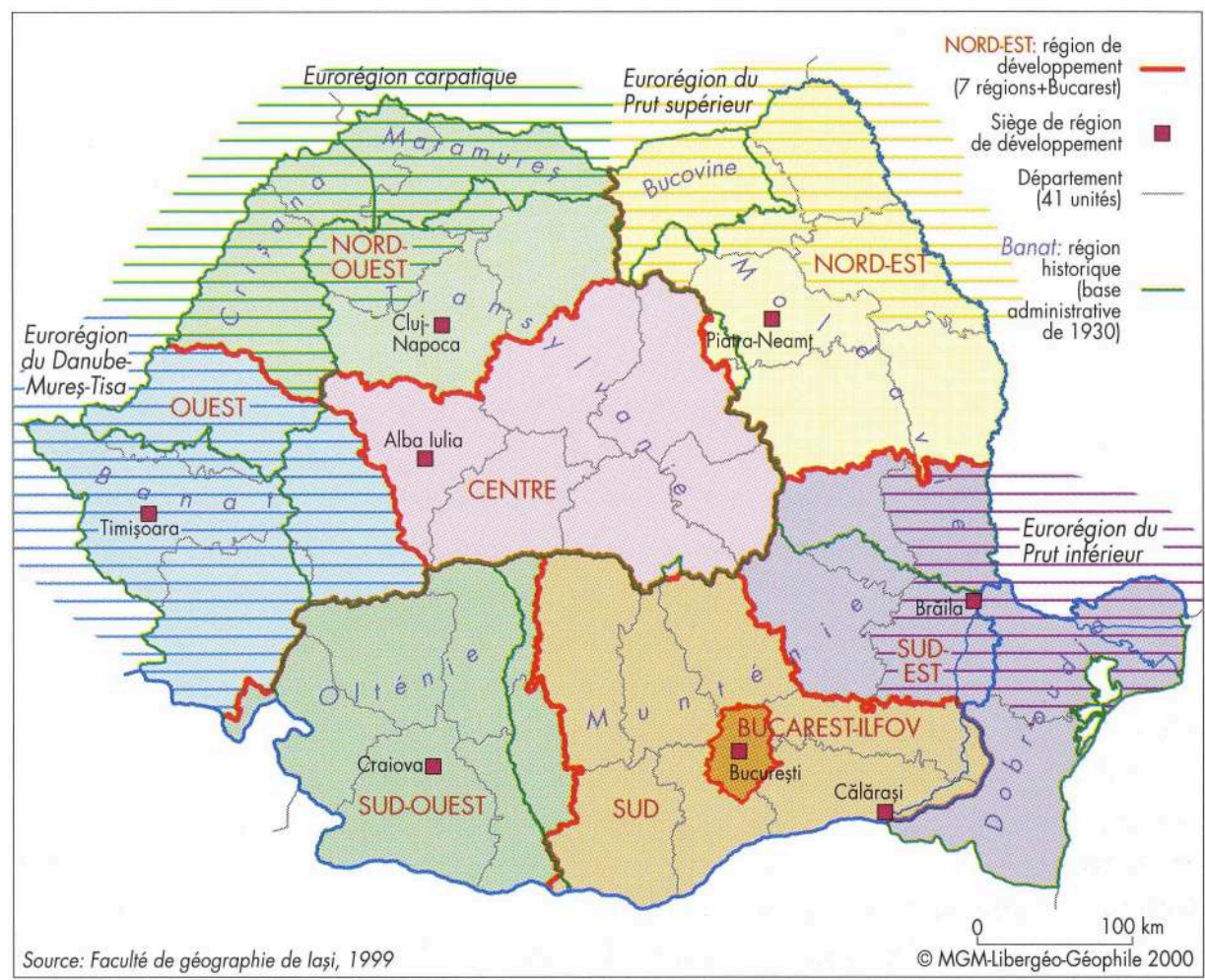

Rey (Violette), Groza (Octavian), lanoş (Ioan), Pătroescu (Maria), Atlas de Roumanie, Paris : CNRSLibergéo-La Documentation Française, 2000. Reproduction avec l'aimable autorisation des auteurs.

Călăraşi, ville moyenne parmi les plus petites, aux marges sud du pays, au dynamisme économique et urbain loin derrière celui de villes comme Piteşti, Târgovişte ou Ploieşti, à l'industrialisation précoce ${ }^{22}$ et en position géographique plus centrale dans la région et le pays, a été désignée, contre toute attente, chef-lieu de la région de développement sud Muntenia qui regroupe sept judeȚ: Ialomiţa, Prahova, Dambovị̧a, Arges, Teleorman, Giurgiu et Călăraşi. Plusieurs explications sont avancées.

La position de la ville et les relations qu'elle entretient avec la Bulgarie ont été déterminantes dans ce choix, selon le responsable du Bureau de la Coopération Transfrontalière ${ }^{23}$, le directeur départemental de la culture, du patrimoine et des cultes qui est également directeur du "Journal de Călăraşi ", énonce l'idée que les bonnes relations de l'ancien président du conseil de judeŢ au Conseil de l'Europe à Strasbourg ont emporté la décision. Les élus locaux du judeŢ de Călăraşi se trouvent ainsi dans une position forte mais qu'ils doivent défendre vis-à-vis des autres collectivités. Cette désignation est une occasion extraordinaire pour la ville et le judeŢ de relancer leurs économies afin de participer à l'intégration européenne et à la mondialisation dans de meilleures conditions. Cette chance d'une revalorisation du statut de la ville et du judeT n'est pourtant pas perçue et saisie par les édiles locaux. Le nouveau statut est, selon le maire de Călăraşi, peu utile à la ville si ce n'est en termes d'image. Les responsables du judeŢ, l'ancien et l'actuel président du conseil, quant à eux, n'en mesurent pas l'intérêt, au contraire, ils négligent de se présenter aux réunions du Conseil de la région de 
développement, faisant courir à la ville le risque de perdre l'implantation du siège central de l'ADR sud Muntenia. Ceci est d'autant plus difficile à comprendre que les partenaires de la région ont été consultés et ont participé à des groupes de travail pour définir la stratégie de développement régional. Préparé par l'ADR aidée du «Département pour le Développement International» de Grande Bretagne, ce document ${ }^{24}$ a été élaboré en continuité de la stratégie d'adhésion de la Roumanie à l'Union européenne sur la base de données régionales. Sans continuité historique et dotées de noms neutres ${ }^{25}$, il est intéressant à ce propos de noter que la région Sud a été rebaptisée Sud-Muntenia pour mieux coller à l'ancien province de l'entre-deuxguerres, mais ni l'une ni l'autre n'appartiennent à l'univers territorial des élus.

\section{Les niveaux intermédiaires du județ et de l'eurorégion, échelon où l'initiative individuelle l'emporte sur le portage collectif des projets}

L'échelon du judeT est bicéphale, la préfecture et les services de l'administration départementale d'une part et le conseil de judeŢ d'autre part. Ces deux entités sont conduites à travailler ensemble pour élaborer un plan pluriannuel de mise à niveau du judeȚ avant l'adhésion à l'Union européenne. Il se présente sous la forme d'un ensemble de six mesures portant sur des objectifs généraux à caractère politique et économique et se décline en programme annuel, celui de 2005 mentionne des thèmes très variés. Néanmoins, celui del'emploi est le thème mobilisateur des autorités locales. Le contexte économique très difficile focalise la préoccupation des édiles sur la venue d'investisseurs étrangers afin de résorber le chômage, de fixer une population active qui regarde vers l'occident et les grandes villes roumaines et d'aider à l'équipement des communes par de nouvelles rentrées de taxes locales. Ce souci se retrouve dans les propos du préfet qui, à une question sur les projets en cours, nous confie un document intitulé "La stratégie de promotion du judeŢ de Călăraşi auprès des investisseurs étrangers" ${ }^{26}$ mis au point dans le cadre du plan départemental traitant de la politique d'intégration européenne. Ce principal objectif de l'emploi n'ouvre pas de réflexion, globale et sur le long terme, portant sur le développement et l'aménagement du territoire du judeŢ. La difficulté ressentie pour mettre en œuvre une cohérence et un suivi des projets peut s'expliquer par la réalité récente de collectivités territoriales indépendantes du pouvoir central et par l'absence d'une tradition régionale en Roumanie.

N'ayant pas dans les régions d'interlocuteurs traditionnels, le pouvoir central a fondé le développement sur le principe du partenariat avec tous les acteurs locaux, ce qui se traduit par une multiplication des initiatives et des réponses aux appels d'offres sans lien les unes avec les autres. Cette situation reflète une absence de coordination entre les acteurs locaux car, au-delà de la planification théorique, les relations interpersonnelles priment. Les élus du judeŢ se font un devoir de prendre des contacts avec des investisseurs étrangers pour les inciter à s'installer dans le département et surtout dans leur commune. Le sénateur PNL(parti national libéral) est en contact très étroit avec l'agence nationale qui collabore avec les investisseurs étrangers et il a réussi à convaincre une entreprise portugaise de biodiésel de s'installer sur sa commune de Lehliu. Le choix du site de Călăraşi pour la construction d'une usine Saint-Gobain de verre plat est un autre exemple, il s'est opéré sur des critères géographiques mais 
l'intervention d'un sénateur PSD (parti social démocrate) a été déterminante pour le choix final de Călăraşi en concurrence avec deux autres sites roumains.

Les difficultés de l'eurorégion du Bas Danube pour exister témoignent des obstacles qu'un projet partenarial doit franchir. A la date d'adoption des statuts ${ }^{27}$, le 15 décembre 2001, elle regroupe du côté roumain trois judeŢ : Călăraşi, IalomiŢa et Constanţa et du côté bulgare, les villes de Dobrič et de Silistra ainsi que l'association de collaboration transfrontalière et de développement " Dobrogea Dunăreană " composée des régions de Silistra et Dobrič.

Figure 5. L'eurorégion du Bas Danube

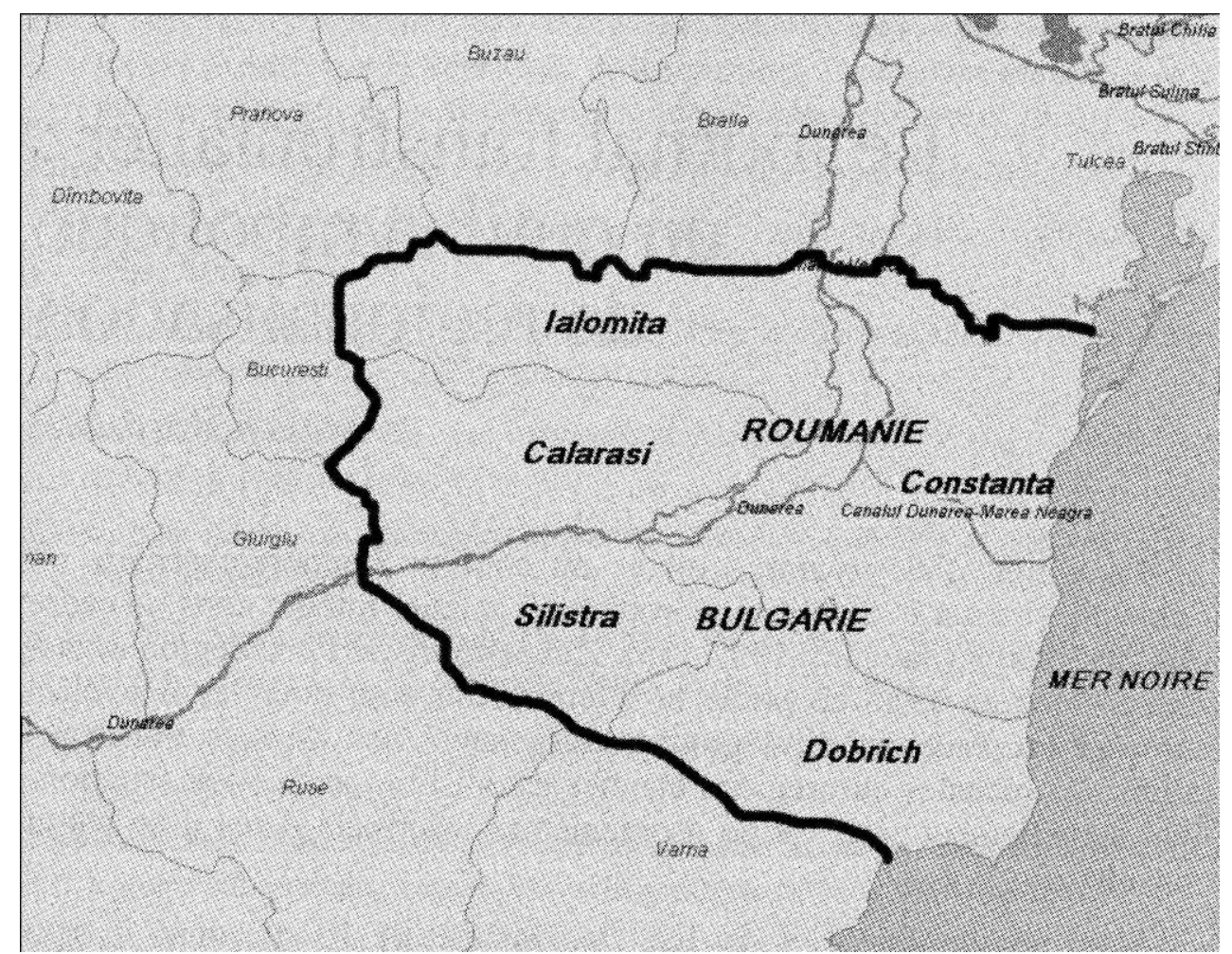

Carte extraite de Bratosin (Stefan), op.cit. Avec l'aimable autorisation de l'auteur

Elle n'est pas une entité supranationale ou supraétatique mais un cadre pour faciliter la coopération interrégionale entre ses membres. Le conseil de l'eurorégion est l'organe de direction, quatre commissions travaillent dans les domaines du tourisme, des affaires, de l'environnement, et du social, et un secrétariat assure la coordination entre le conseil et les commissions. Elle ne fait pas l'unanimité quant à son utilité et ses résultats. Bien que le préfet affirme qu'il n'y a pas de frein à la coopération entre la Roumanie et la Bulgarie, d'autres acteurs la considère comme une "coquille vide" qui n'a à son actif qu'une succession de petits projets entre 10000 et $50000 €$ sans envergure régionale et sans lien entre eux, telle que l'installation à Călăraşi de petits dos d'âne routiers. Ne pouvant assurer une continuité des missions, c'est le personnel des judeţ qui se charge des dossiers de l'eurorégion.

L'ancien président du conseil de judeţ qui fut le premier président de l'eurorégion est à l'origine de sa création en 2001 ; il maintient de bons contacts avec la ville de Silistra mais selon l'opinion du responsable du Bureau de la Coopération Transfrontalière, 
l'eurorégion ne fonctionne plus depuis le changement d'équipe du conseil de judeŢ. Les intentions sont là, les projets sont annoncés mais les réalisations attendent plusieurs années, quand elles ne sont pas annulées tout simplement par manque de mobilisation pour la recherche de partenaires financiers. Le projet de construction d'un point de passage de la frontière entre Călăraşi et Silistra consiste en la construction d'un nouveau poste de douane à Călăraşi, la réfection de la route y menant et l'achat d'un ferry-boat. Il date de 1993 et a reçu le soutien financier du programme PHARE CBC en 2001 et fin 2006 il n'est toujours pas achevé. Il est l'objet de querelles incessantes entre les deux partis politiques dominants dans la ville, le PSD et le PNL, qui ont été successivement à la tête du judeȚ et qui se rejettent la responsabilité du retard du projet. Un manque d'implication, une mauvaise circulation des informations laissent dans l'ombre des initiatives destinées pourtant à la promouvoir, c'est le cas du journal "Dunărea de est", journal de l'eurorégion paru en 2004. Il est écrit en roumain par l'association bulgare "Dunărea Dobrogeană" et relate certains événements liés à l'eurorégion, aux programmes européens et propose des articles sur les localités roumaines et bulgares de l'eurorégion. La publication de ce journal a été financée la première année, en 2004, sur des fonds européens, il devait être distribué aux institutions puis au public. En 2005 il n'existe plus et ni le sénateur PNL ni le directeur départemental de la culture, du patrimoine et des cultes n'ont eu connaissance de l'existence de ce journal.

\section{Un projet urbain fonctionnaliste}

Enfin, à l'échelon communal, une situation comparable s'observe. Le plan d'urbanisme ${ }^{28}$ de 1995 envisage le développement de la ville selon des modalités fonctionnalistes (emploi, habitat, équipements...) et non spatiales. Il se propose d'éliminer les dysfonctionnements sans considérer l'espace urbain comme un tout dont le fonctionnement doit être analysé. Ainsi, pour la zone centrale, sont énumérés les problèmes à résoudre comme la dégradation des constructions, les problèmes de circulation aux intersections de certaines rues, l'absence de parking en limite de la zone piétonne et commerciale, l'insuffisance du réseau d'éclairage public, le sousdimensionnement des canalisations existantes etc. Par exemple, la promenade sur les berges du Borcea est jugée attirante pour son calme et pour accroître l'agrément des lieux il est proposé d'y construire un bassin couvert pour accueillir les compétitions sportives, les habitants et les touristes. Ce fonctionnalisme se traduit par l'absence de réflexion sur les liaisons entre la berge et les quartiers voisins, l'accès à celle-ci et au bassin, le stationnement et la visibilité du Borcea depuis le centre-ville. À l'heure actuelle, la promenade n'est pas mise en valeur, on la découvre par hasard. Elle n'a pas d'intérêt en soi, elle est très petite et sans aménagement particulier, c'est davantage le jardin public qui longecelle-ci, qui apporte une note agréable au lieu.Cette appréhension du devenir de la ville se retrouve dans la profession de foi du maire de Călăraşi ${ }^{29}$ qui se présente sous la forme d'une liste d'actions ponctuelles à réaliser, du type renouvellement des canalisations de telle rue ou réfection de la chaussée d'une autre.

34 Une réflexion par trop fonctionnaliste est préjudiciable à une bonne coordination entre les différents niveaux décisionnels. On ne constate pas de synergie entre eux et le lent mouvement de décentralisation ne permet pas encore la mise en place du principe de subsidiarité. En 2005 les berges du Danube appartiennent toujours à l'Etat empêchant la 
commune de réaliser les aménagements qu'elle souhaite. La décentralisation n'a pas non plus généré un pouvoir local fort, capable de prendre à son compte certaines revendications qui le concerne directement. Le relais doit être assuré par d'autres personnalités. Ce fut le cas lors de la construction de la bretelle autoroutière menant de l'autoroute Bucarest-Constanţa à Călăraşi puis jusqu'au point de passage sur le Danube, qui a suscité peu d'intérêt localement. Il a fallu une mobilisation de la presse et du Sous-préfet pour obtenir le démarrage des travaux d'une infrastructure financée par l'Union européenne et l'Etat roumain. Cette infrastructure place pourtant Călăraşi dans une position très attractive, avec une liaison rapide entre les deux principales villes de Roumanie et avec Silistra, propice à un développement fructueux entre les partenaires de l'eurorégion.

Cette absence de vision d'ensemble et à long terme, de la part des politiques et la difficile perception du lien et de la hiérarchie entre tous ces échelons, obscurcissent encore l'action de ceux-ci pour la population qui doute en l'avenir de la ville et du judeT et juge qu'il y a peu de changement positif. Pour les quelques personnes interrogées, la ville a un potentiel, celui du Borcea, mais tout est encore à faire pour le mettre en valeur. Cependant des initiatives individuelles voient le jour dans des domaines qui abordent le développement sous un angle nouveau, l'environnement et le tourisme, ou s'appuie sur une tradition locale qui a survécue au communisme, la culture. Si les projets touristiques en restent encore au stade des bonnes intentions, les initiatives en faveur de l'environnement sont plus concrètes, mais c'est surtout par ses manifestations culturelles que Călăraşi se distingue et acquiert une notoriété qui dépasse largement les limites du judeŢ.

\section{Une timide relance de la dynamique danubienne}

\section{Călăraşi : une ville de tradition culturelle}

Alors qu'il est reproché à la population de peu s'impliquer dans les partis politiques qui éprouvent des difficultés à recruter des personnes de valeur, la participation aux manifestations culturelles qui animent la ville de Călăraşi tout au long de l'année est massive. Le Danube y retrouve une place importante, il fait l'objet d'expositions de photos sur les paysages du fleuve ou d'exposition portant sur les activités traditionnelles qui s'y pratiquent comme la pêche. Il a en effet toujours été fortement associé à l'histoire de Călăraşi et Silistra comme axe de développement pour leur hinterland. Il est aussi le symbole fort de la construction européenne, en tant que grand fleuve il fut à la fois axe de communication, de conquête, vecteur de la culture européenne depuis l'occident jusqu'à l'orient du continent. "Il donne sens à l'espace" 30 , celui-ci même que l'Europe, après les années de rupture, cherche à reconstituer au travers d'une politique internationale de coopération que l'on voudrait facteur d'intégration ${ }^{31}$ en particulier à l'échelle des régions transfrontalières. La population est davantage sensible à la renaissance des relations transfrontalières en souvenir de l'époque où Bulgares et Roumains vivaient ensemble de part et d'autre de la frontière. Cette période prend fin avec le traité de Craiova (7 septembre 1940) qui rétrocède à la Bulgarie les deux départements du sud de la Dobroudja (Silistra et Dobrič) et dont la mise en œuvre s'accompagne d'échanges de populations. L'enthousiasme se manifeste aujourd'hui par la multiplication des relations transfrontalières dans le domaine de la culture, mais en général, les initiatives relatées dans les journaux ne mentionnent pas 
de liens avec l'eurorégion proprement dite. C'est le cas du festival mondial de caricatures de Călăraşi installé à la maison de l'humour de Gabrovo, en Bulgarie. D'autres initiatives transfrontalières ne relèvent pas de l'eurorégion. Il en est ainsi des musées roumains et bulgares des villes riveraines du Danube (Giurgiu, Ruse, Tutrakan, OlteniŢa, Călăraşi, Silistra, Dulovo, Mangalia, Constanţa, Varna et Dobrič) qui ont conclu en 2006 un protocole de coopération pour organiser, par exemple entre olteniţa et Tutrakan, des échanges d'expositions, un circuit touristique commun ou participer ensemble aux fêtes locales, revivifiant les coutumes locales pratiquées il y a moins d'un siècle sur les deux rives du Danube.

\section{La préoccupation de l'environnement est encore une affaire de spécialistes}

Malgré les efforts fournis par la Bulgarie et la Roumanie pour rattraper le retard vis-àvis de l'Europe, l'attitude du "chacun pour soi" se ressent dans la mise en place du volet environnemental du développement durable dont l'efficacité se mesure sur des étendues qui dépassent les frontières. La déclinaison de ce concept est une obligation de l'Europe et les pays candidats ne peuvent s'y soustraire. Sa mise en pratique est du ressort des administrations et services publics dont la motivation rencontre souvent l'opposition ou le manque de volonté, héritage d'un passé proche. En d'autres termes, la forme prime sur le fond, les études d'impact sont réalisées parce qu'elles sont devenues obligatoires pour répondre aux exigences de Bruxelles. La conviction des agents des services concernés par la protection de l'environnement et du cadre de vie emporte parfois l'adhésion des autorités. Le projet d'installation de deux stations de surveillance de la qualité de l'air à Călăraşi et Silistra a été terminé en 2002 et celui sur l'agriculture biologique mobilise les partenaires roumains et bulgares en 2003 pour former les fermiers de Călăraşi et de Silistra à de nouvelles pratiques et créer un réseau d'échange d'informations. L'Agrocenterbusiness de Silistra, à l'initiative du projet, a travaillé en collaboration avec l'institut de recherche de Fundulea, reconnu dans ce domaine dans toute la Roumanie. Malgré cela, un certain attentisme persiste, les responsables locaux et la population ne mettent pas beaucoup d'empressement à faire valoir leurs droits sur les berges du Danube pour une mise en valeur de celles-ci, pourtant la marque de leur identité danubienne. La création d'une réserve naturelle à Ostrov entre le Danube et le Borcea, des projets de coopération pour la protection des berges du Danube et la conservation des zones humides des lacs du département, sont des projets du judeT dont on ne parle plus comme des réalisations prochaines. Cependant, la nature et les paysages des rives du Danube, chers au coeur des habitants de Călăraşi sont les images retenues par tous pour promouvoir Călăraşi et cette région de Roumanie.

\section{La stratégie de promotion du tourisme par la valorisation de l'image du Danube}

Pour les habitants de Călăraşi comme pour les Roumains, le Danube qui longe la Roumanie sur $1074 \mathrm{~km}$ représente le pays. Très présent dans la vie des habitants de Călăraşi, il donne son nom à des associations : une coopérative d'artisans, une équipe de football etc. Il est le sujet de rencontres, celle qui a eu lieu en 1999 à Călăraşi au 
Centre de la culture européenne entre jeunes Français, Italiens et Roumains avait pour thème "Le Danube, vecteur de la civilisation européenne". Tout ce que représente le Danube comme souvenirs historiques, support d'une culture et symbole d'une nature encore sauvage, nourrit de grands espoirs pour la région de Călăraşi. Chacun reconnaît les potentialités touristiques de la ville et du judeŢ. Dès 2001, les maires d'OlteniŢa et de Călăraşi avec le soutien du conseil de judeT, de la préfecture et des parlementaires des deux villes, se sont mobilisés pour promouvoir le tourisme sur le Danube au salon du tourisme de Romexpo à Bucarest. En se projetant dans l'avenir, on retrouve le thème du tourisme dans le programme départemental de 2005, il est identifié comme un secteur économique avec un potentiel de développement représenté essentiellement par le Danube. Les efforts de communication et de promotion des possibilités touristiques qu'offre le Danube se poursuivent. La plaquette du département vante les possibilités de pêche et de chasse, la beauté des paysages qui se découvrent à la vue des passagers de bateaux de croisières, le site internet de la préfecture présente les paysages du Danube. L'accent est mis sur le tourisme d'affaires, pourvoyeur de rentrées financières importantes. Le concept du "Danubius park" de Călăraşi prévoit des infrastructures touristiques (musée ethnographique, centre équestre, boutiques d'artisanat, aménagement pour randonnées, port de plaisance ...) et d'affaires (centre de conférences et de rencontres, hôtels...) sur le modèle des parcs industriels. Il s'adresse en priorité à une clientèle étrangère qui retrouve là les attractions standardisées des lieux touristiques artificialisés des pays occidentaux, assez éloignés de la culture locale et des paysages du fleuve. Encore une fois, par manque de planification le projet n'a pas encore abouti : problème de terrain à échanger avec Romsilva ${ }^{32}$, études d'environnement inachevées. Le manque de continuité d'une équipe à l'autre se ressent sur ce thème qui pourtant fait l'unanimité. L'ancienne équipe dirigeante du județ avait mis au point une stratégie de développement du tourisme rural le long du Danube par la mise en valeur de lacs et de réserves naturelles, accessible par une nouvelle route. La nouvelle équipe ne se l'est pas appropriée et reprend des études devant permettre d'élaborer une stratégie de développement du tourisme avec les Bulgares.

\section{Conclusion}

Au travers du cas de la ville de Călăraşi et de ses relations au Danube il apparaît que ce dernier n'est pas encore redevenu l'axe de développement des régions riveraines de Roumanie et de Bulgarie. Il reste néanmoins le symbole d'une culture vivante qui rassemble les habitants des deux rives. Le développement économique et social des régions de frontière requiert un engagement fort des Etats. Or le Danube pâtit d'une part, d'une faible coopération entre les deux pays, ceux-ci ayant décidé de rejoindre l'économie de marché et la démocratie chacun de leur côté et non en collaborant pour relever les défis de l'intégration européenne ensemble. D'autre part une réforme de la décentralisation engagée depuis peu de temps ne met pas les élus locaux en situation de décider et d'agir. On remarque néanmoins une nouvelle dynamique endogène. Des initiatives ponctuelles, en matière de développement de l'agriculture biologique, pourraient se développer. L'identité danubienne est bien présente dans la population et est entretenue depuis le plus jeune âge : de nombreux projets de coopération avec la Bulgarie proviennent des écoles. Les décisions des Etats de l'ancien régime n'ont pas éteint la mémoire des lieux qui reprend vie dans la société civile, celle-ci devance le 
monde politique encore sous l'emprise des méthodes de l'ancien régime et contraint par les moyens financiers exsangues des collectivités territoriales et de l'Etat. Il faut encore donner le temps à la société roumaine de renouer avec la démocratie et d'assimiler les principes de la démarche de projet.

\section{NOTES}

1. 1992 : 76952 habitants ; 2002 : 70039 habitants, Institutul NaŢional de Statistică - Direcţia Regională de Statistică Călăraşi, .SituaŢia demografică a judeŢului Călăraşi 1993-2003.

2. Tudor (Constantin) et Vlădilă (Petre), Aspecte ale evoluŢiei aşezării de la cotul Borcei (Călăraşi) in perioada 1541-1821 [Aspects de l'évolution de l'agglomération du coude du Borcea (Călăraşi) entre les années 1541-1821], Cultura si civilizatie la Dunarea de jos, II, 1986, pp. 239-246.

3. Primăria Municipiului Călăraşi, monografie, Călăraşi : Agora, 2003.

4. Ritter (Jean), Le Danube, Paris: PUF, 1976.

5. Primăria Municipiului Călăraşi, op. cit.

6. Zbîrnea (Ileana), La citta di Călăraşi - premesi dello sviluppo urbanistico nel XIX secolo [La cité de Călăraşi - Les premiers développements urbains au XIX siècle], Cultura si civilizatie la Dunarea de jos, XII-XIV, 1995, pp. 335-346.

7. Texte recueilli à Ciocaneşti.

8. Vlahuta (Alexandru), Romania pitoreasca [La Roumanie pittoresque], Bucarest, Minerva, 1972, p. 39-40.

9. Pământul, 20-26 septembre 1997.

10. Primăria Municipiului Călăraşi, op. cit.

11. Rădulescu (Florin), « Istoria oraşului în hărŢi » [L'histoire de la ville dans les cartes], Campia, $\mathrm{n}^{\circ} 4,2006$, pp. 20-21.

12. Rădulescu (Florin), op.cit.

13. Rădulescu (Florin), op.cit.

14. Di Méo (Guy) Géographie sociale et territoire, Paris: Nathan, 1998.

15. Chassereau (Aude), Peyon (Jean-Pierre), « Le projet île de Nantes ou comment la ville se réconcilier avec son fleuve», actes du festival de géographie de Saint Dié, http://fig-stdie.education.fr/actes/actes_2003/dussoy/article.html

16. Ibidem.

17. Salles (Sylvie), "Le rapport à l'eau dans les projets d'aménagement urbain », actes du colloque de Saint Dié, http://fig-st-die.education.fr/actes/actes_2003/dussoy/article.html

18. Ianoş (Ian), Tălângă (Cristian), Braghină (Cristian), Vîrdol (Amalia), "Romania-Bulgarian cross-border cooperation. Reality and wishes " [Coopération transfrontaliere Roumano-Bulgare. Réalités et souhaits], Revue roumaine de géographie, Tomes 43-44, pp. 73-83, 1999-2000.

19. Bratosin (Stefan), "Le journal transfrontalier : un outil symbolique de régionalisation ", Communication, 25 (1), 2006, pp. 195-204.

20. Sanguin (André-Louis), «Formation étatique, frontières et nationalités en Europe médiane » Maurel (Marie-Claude), Recomposition de l'Europe médiane, Paris, SEDES, 1997.

21. Groza (Octavian), « Maillages administratifs officiels et identités territoriales officieuses : les échelons spatiaux de la différenciation identitaire en Roumanie » in Rey (Violette) et Saint Julien (Thérèse) (dir), Territoires d'Europe. La différence en partage , Lyon : ENS Editions, 2004, pp. 195-206. 
22. .Ibid.

23. ONG basée à Călăraşi, elle a pour mission de gérer les fonds transfrontaliers pour les trois ADR Sud Muntenia, Sud-Est et Sud-Ouest Oltenia.

24. Strategia regionala Sud Muntenia 2004-2006.

25. Groza (Octavian), Muntele (Ionel), «Le maillage sans territoire : la région de développement en Roumanie " in Rey (Violette), Boulineau (Emmanuelle) et Coudroy de Lille (Lydia) (coord.), L'élargissement de l'Union européenne : réformes territoriales en Europe Centrale et Orientale, Paris : l'Harmattan, 2004, pp. 195-206.

26. Institutia prefectuluijudeŢul Călăraşi, Strategia de promovare a investitiilor straine pentru județul Călăraşi [Stratégie de promotion du judeŢ Călăraşi auprès des investisseurs étrangers], octobre 2005.

27. Conformément à son article 3 , sa composition n'est pas définitive et d'autres unités administratives de Roumanie et de Bulgarie peuvent devenir membre.

28. Ministerul Lucrarilor Publice şi Amenajarii Territoriului : Urbanproiect, Plan urbanistic zonal : zona centrala Calarasi, vol 2 : Memoriu generala, 1995. Le plan d'urbanisme en cours de révision lors de notre visite en 2005 n'a pu être consulté.

29. Pour les élections municipales de 2004.

30. Dussoy (Gérard), «Eau et espace politique: voies navigables et intégration territoriale des grands espaces ", actes du colloque de Saint Dié, http://fig-st-die.education.fr/actes/actes_2003/ dussoy/article.html

31. Ibid.

32. Office de gestion de la forêt domaniale.

\section{RÉSUMÉS}

L'étude du rapport au Danube de la ville de Călăraşi est à replacer dans le contexte de la transition vers la démocratie. Călăraşi, contrairement aux villes occidentales, est toujours dans une phase d'industrialisation, celle-ci, conjuguée à une extension urbaine parallèle au fleuve, ne favorise pas les relations ville-fleuve et tend à relayer ce dernier au rang des souvenirs d'enfance. La tradition d'un pouvoir centralisateur, sans relais local fort, nuit à la mise en œuvre d'un aménagement global et cohérent promu par l'Europe. Le manque de coordination qui s'ensuit et la faible coopération avec la Bulgarie ne sont pas favorables à des actions d'envergure sur le Danube et ses riverains éloignent de celui-ci.

The study of the relationship in the Danube of the city of Călăraşi is to be replaced in the context of the transition towards the democracy. Călăraşi, contrary to the western cities is always in a phase of industrialization, this one conjugated to an urban extension parallel to the river don't promote the relations city-river and so much to relieve this last one to the rank of the memories of childhood. The tradition of a power advocate of centralizing, without strong local relay, harms in the implemented of a global and coherent development promoted by Europe. The lack of coordination which follows and the weak co-operation with Bulgaria are not favourable to largescale actions on the Danube and take away this one of his local residents. 
INDEX

Index géographique : Danube, Roumanie, Călăraşi

Mots-clés : Développement régional, Coopération transfrontalière, Urbanisme

\section{AUTEUR}

ODILE BRATOSIN

Doctorante à l'ENS-LSH au laboratoire Géophile, UMR 5600 bratosin_odile@yahoo.fr 